\title{
ENVIROFI - Bringing Biodiversity to the Future Internet
}

\author{
Katharina Schleidt ${ }^{1}$, Nina Laurenne ${ }^{2}$, Andrea Giacomelli ${ }^{3}$, and Denis Havlik ${ }^{4}$ \\ ${ }^{1}$ Umweltbundesamt Austria GmbH \\ katharina.schleidt@umwel tbundesamt.at \\ ${ }^{2}$ Semantic Computing Research Group (SeCo), Aalto University \\ firstname.lastnamedaalto.fi \\ ${ }^{3}$ Consiglio Nazionale delle Ricerche \\ a.giacomelli@iia.cnr.it \\ ${ }^{4}$ Austrian Institute of Technology \\ Denis.Havlik@ait.ac.at
}

\begin{abstract}
In order to meet the requirements for observational biodiversity data, new sources of data must be enabled; for this purpose, new tools will be required. In order to effectively implement such tools, standardized building blocks such as the enablers defined by the FI will be very valuable. Thus, it is of utmost urgency that the requirements posed by the biodiversity sector are clearly structured and made available for implementation of environmental enablers within the FI. The ENVIROFI project has gone to great lengths so provide these requirements for various environmental domains; of specific relevant to this paper being the biodiversity domain. We hope to successfully contribute to the future of e-Environment in this manner.
\end{abstract}

\section{Introduction}

In the last decade, the pervasiveness of the Internet has become such that a world without online access to available resources has become unthinkable. At present, the internet consists of loosely linked documents and web applications. The Future Internet (FI) [1], an initiative of Organisation for Economic Co-operation and Development (OECD), seeks to expand this to the internet of People, Things and Services.

Information and communication technologies (ICT) for the environment generate huge amounts of environmental observations. At the same time, FI technologies and mobile applications empower amateur naturalists to provide as well as consume data. These data must be harmonized, exchanged between various stakeholders and processed - often in real time.

The ENVIROFI project, funded by the European Commission under the Seventh Framework Program (FP7) Future Internet Public Private Partnership (FI-PPP) Programme, addressed the needs and use cases of the environmental usage area within the FI-PPP context.

The project envisions a European Observation Web for the environment enabled by the FI. With this in mind, it explored the next generation of an ICT infrastructure which will enable global, decentralized, interactive, and dynamic environmental applications; integrating the data, services, and models from governments, the private sector, and other observation data. 
Within the ENVIROFI project, Work Package 1 "Bringing Biodiversity into the Future Internet" focused on the use of Future Internet technology for survey, analysis, quality assurance, persistence and dissemination of biodiversity data in the following areas:

- Requirements for mobile data entry devices for recording biodiversity occurrence data.

- Requirements for context aware quality assurance of reported data, automated identification support and intelligent crowdsourcing of biodiversity occurrence data.

- Standards based data models for observational data as well as a semantic backbone for biodiversity data.

This paper analyses the requirements to the Future Internet of a mobile biodiversity occurrence information system, and proposes solutions required for the creation of such a system.

\section{Requirements on Mobile Devices}

\subsection{Non-functional Requirements}

As an initial step, an analysis of the requirements for mobile data entry devices for recording biodiversity occurrence data was performed. Traditionally, biodiversity data stems from organized surveys performed by trained professionals.

In many cases the results of the data survey are initially collected in paper logbooks and then later transferred to electronic media due to constraints of using mobile devices in the field. One of the reasons for the reluctant uptake of mobile devices was the prohibitive cost of professional devices such as the handheld devices created by companies such as Trimble ${ }^{1}$. Through the advent of powerful and inexpensive mobile devices, it is now possible to create affordable solutions for mobile biodiversity data entry.

Due to the increased financial pressures on the environmental sector, it is often not possible to obtain the necessary amount of observational data by relying only on professional survey data; thus, this data must be complemented by data provided by experienced amateur naturalists. While these potential data providers often possess a great deal of knowledge over certain areas of biodiversity, they are less likely to invest large amounts of time and energy in repetitive activities such as performing fledged professional surveys. Consequently, these users must be offered a possibility to report only part of the required observational data and encouraged to improve the overall survey quality at their own pace. Ideally, the same application should be presented in various levels of complexity for different user groups, using simpler terms and requiring fewer types of observations for less experiences users.

Data provision should be bidirectional, with the user able to interactively obtain information about habitats and relevant biodiversity in a specific area. In addition to this

http: //www.trimble.com/ 
being a strong support for professional users in their work, this opens the scope of the mobile applications for a wider community of amateurs, allowing them to gain valuable information on the area of interest.

As many relevant areas of interest for the biodiversity sector are remote areas, one cannot assume that the mobile application will have constant connectivity. The application must remain fully functional during the duration of a field trip and opportunistically exchange information with the central database in the areas with good network coverage. This means that:

1. The user must be able to select a specific area in which they will be working or trekking in advance while they still have full network connectivity and all relevant data for this area must be cached for later use when in the field.

2. All observational data provided by the user in the field must be stored locally and uploaded once network connectivity is available.

3. The feedback on observations entered by user (e.g. assessing of the plausibility by other users or background services) can only be provided asynchronously.

A further difficulty often encountered during protracted use of mobile devices is the battery duration. Using current technology only about 4 hours of continuous use are possible, severely constraining the scope of use for mobile applications. While the mobile application can be designed to keep energy requirements to a minimum, the techniques used often interfere with the usability of the system; thus, we are hoping that new technological developments will provide a new generation of devices with the required battery duration as required for professional field work.

\subsection{Main Usage Patterns}

The biodiversity survey applications must support following types of use

- Output:

(a) View locations of existing species and habitat occurrence records;

(b) View detailed information on observations on existing species and habitat occurrence records;

(c) View further information on species or habitats (nice-to-have).

- Input

(a) Provide a new species or habitat occurrence record with location;

(b) Provide new observations on existing species and habitat occurrence records;

(c) Support functionality for species and habitat identification.

- Administrative

(a) Define and cache areas of interest for later offline use in the field;

(b) Download species and habitat occurrence data;

(c) Provide feedback on existing and new species or habitat occurrence records and observations;

(d) Standardized data models for access to species or habitat occurrence records and observations;

(e) Standardization of species and habitat nomenclature. 
These usage patterns are found in all participatory sensing applications, but some adaptation will be required for an optimal user experience on mobile devices.

\section{Quality Assurance of Biodiversity Observations}

Quality assurance of biodiversity data has always been a challenge. In contrast to other areas of environmental data provision, where the focus is on physical and chemical measurements, biodiversity data has always depended on observational data provided by humans, with all subjective errors entailed. While in some cases these observations are complemented by physical specimens that can be archived for future reference, there is a multitude of reasons why this is not often possible, including:

- endangered species should not be further endangered by removal of specimens, be they entire individuals or only parts (i.e. leaves or branches);

- certain species are dangerous, and thus should only be collected by professionals;

- some species can only be observed at a distance.

In addition, storage of specimens is costly. Many institutions currently tasked with the storage and conservation of scientific specimens are struggling to maintain their current collections; some have been closed, with other institutions struggling to find storage opportunities for the existing material. Thus, adding vast amounts of additional specimens as would result from wider data provision to these already overburdened institutions is not an option. New mechanisms for assuring the correctness of field observations must be found.

To date, biodiversity occurrence data is provided with one unique species identification. Upon later inspection of the data, this identification is often shown to be wrong; a new identification is provided replacing the old one. However, there is no guarantee that the new identification is actually correct; by removing the old identification originally provided, valuable information can get lost. Thus, we propose allowing for the provision of multiple identifications for one species occurrence record, whereby each species identification is assigned a level of plausibility. While the species identification with the highest plausibility will be displayed to the user as the default identification, it should be possible to view the other identifications provided if desired.

\subsection{Context Aware Quality Assurance}

One approach for assuring the quality of biodiversity observation data is a context aware approach. Through the mobile devices used for the provision of biodiversity observations, the system can report the exact location of the user at the time of reporting the observation, as well as the time at which the observation was first reported to the system.

By comparison of the species reported by the user with existing background information such as known species distributions a plausibility of the reported species existing in the area reported can be calculated. However, species distribution information is only available for certain species, is not always completely reliable, and is prone to 
change due to additional factors such as human pressures and climate change. Thus, additional spatial mechanisms must be used for the calculation of the plausibility of a biodiversity occurrence record.

Various additional mechanisms identified for extending the possible scope for the calculation of species occurrence plausibility include:

- comparing the biogeographical region the species is known to occur in with the biogeographical region the species observation was reported from;

- comparing the habitat the species is known to occur in with the habitat the species observation was reported from;

- using the distribution of a species known to prefer a similar habitat to determine the plausibility that the reported species could occur in the region reported;

- using Food-Webs together with the distributions of other species known to be linked to the reported species via a food-chain.

In addition to the spatial mechanisms listed above, temporal information can also be valuable in determining the plausibility of a species occurring at a specific place and time. Based on phenological information pertaining to the species reported such as migratory patterns, the date of emergence of leaves and flowers or the date of leaf coloring and fall in deciduous trees, the system can determine the plausibility that the reported species could actually be sighted at the place and time reported.

A further mechanism for determining the plausibility of a specific species occurrence record utilizes knowledge on common mistakes made in species identification. While one would assume that these types of mistakes only pertain to amateur naturalists, systematic misidentifications are also common amongst scientists and often propagated from one generation of scientists to the next. Using local knowledge on common misidentifications, the system can show the user reporting a commonly misidentified species images of the other more plausible species; this can help the user decide on the final identification of the individual organism sighted, as well as providing further plausibility information for the biodiversity occurrence record.

\subsection{Automated Identification Support}

Various types of automated support in the identification of species have been explored both within ENVIROFI as well as in other related projects. Organizations such as NatureGate [2] in Finland provide well organized identifications keys utilizing the power of IT-based systems to allow a user to provide those species characteristics. Institutions such as the Laboratoire Informatique \& Systématique [3] provide generic identification key software [4] that can be configured by providing SDD [5] encoded species characteristics. As this area has been well explored in other projects, it was not directly considered within ENVIROFI; however, such functionality would be essential for a complete production system.

The two areas of automated identification support explored within the ENVIROFI project pertain to the automatic recognition of species based on images or sounds recorded. For the identification of images, as the provision of a species identification based on an entire individual is not currently technically possible, a fairly sound identification can be provided based on the image of an individual leaf of a tree. 
Of these two approaches, image recognition was prototyped pertaining to leaves of trees. Identification services have been implemented that analyze the shape and coloration of a leaf image provided and return a ranked list of possible species identifications. This list of possible identifications can then be provided to the user together with further images representative for this species, allowing the user to decide which of the possible species identifications returned best corresponds to the individual tree they are trying to identify.

While it is possible to identify some species using only leaf images, for the correct identification of many species, further information will be required. In some cases this complementary information could be provided through additional images of other parts of the tree such as flowers or fruits. In other cases more complex morphological information will be required; for this approach it would be advisable to integrate the leaf recognition service with an identification key for the provision of such information.

Regardless of the approach chosen for automated identification support, which can provide valuable support in the identification process, it is imperative that the final decision on the identification of the individual sighted is left to the human user.

\subsection{Crowdsourcing of Biodiversity Occurrence Data}

Traditionally, biodiversity occurrence data stems from organized surveys performed by trained professionals. Due to the increased financial pressures on the environmental sector, the necessary resources are often not available to perform the survey activities required for the comprehensive assessment of the state of biodiversity.

At the same time, with the advent of various networked platforms and social media, grass-roots activities triggered by non-professionals with an interest in a specific area are becoming a widespread alternative to existing organized governmental and scientific activities. This can provide a valuable complement to existing data and information available from conventional sources. At the same time such activities can serve to sensitize the general population to various issues pertaining to biodiversity, both by allowing them to actively engage in the survey process as well as by providing the users with new information directly pertaining to their current interest through context sensitive information provision.

However, when dealing with crowd-sourced data, it is difficult to ascertain the reliability of the data provided. While these potential data providers often possess a great deal of knowledge over certain areas of biodiversity, it is difficult to determine the reliability of the data they provide without some sort of certification of their individual track-record; thus, the approach of storing the user's reliability rating has been selected. In an initial phase, only one reliability rating will be stored together with the user data; however, in production use the reliability rating must be defined at a far more fine-grained level, providing information on the user's level of proficiency pertaining to different areas of biodiversity. Proficiency categories can be defined based on the structure of the taxonomic hierarchy, using the taxonomic ranks Order, Family or Genus for the definition of a proficiency rating. A user having a high proficiency rating for certain plant families may have a very low proficiency rating pertaining to animals. Depending on the species identified, the relevant user rating should be taken into account in determining the plausibility of a species identification. 
An additional factor in determining the plausibility of a species identification can be provided by utilizing the user's own trust in their identification skill. While in many cases the user will be sure of the correct identification they are providing, in other cases the user will be aware of the fact that they are not confident of the identification being provided. By allowing the user to add a subjective confidence rating to their identification, further information on the plausibility of this identification can be obtained.

Various factors can be utilized to determine a user's reliability rating. An initial value can be set depending on the context through which the user has registered with the system. A user registering via an accredited organization or providing proof of higher education in a specific relevant area is provided a higher reliability rating than an anonymous user. However, the user's reliability rating can be subsequently modified depending on the accuracy of data provided by this user; if the identifications provided with observation records by this user are often corrected by subsequent users, the user's reliability rating will be reduced. Conversely, if the identifications provided with observation records by this user are regularly confirmed by more experienced users, the user's reliability rating will be raised.

In order to perform these adjustments on a user's reliability rating, methods of providing subsequent feedback to existing biodiversity occurrence records must be provided. The following options have been proposed:

- Re-identification of existing biodiversity occurrence records in the field: if a user comes across an individual in the field that has an incorrect identification, the user may provide a different identification for this individual.

- Provision of test images at the end of the identification process: once a user has completed the identification process for an individual, the user is shown six existing images, whereby these images fall into three categories as follows:

- images with a high plausibility rating for the species the user just identified;

- images which are definitely not the species the user identified (but may be commonly misidentified as this species);

- images which have a low plausibility rating for the species the user just identified.

If the user correctly selects the images that have a high plausibility rating for the species identified and rejects the images that are definitely not this species, the plausibility that the new observation provided is correct can be raised. At the same time, if the user has shown proficiency in the identification of this species based on the known material, their choices pertaining to the occurrence records with a low plausibility rating can be used to either raise or lower the plausibility for these occurrence records as well.

- Feedback via a web portal: while mobile devices are very useful for user interaction in the field, their use is limited due to the small display size as well as difficulties in data entry. Thus, a web portal allowing the user to browse through existing biodiversity occurrence records and provide feedback on the occurrence data provided is a valuable complement to the mobile applications that are the focus of this work. 
While there will never be full certainty in the identification of biodiversity occurrence records stemming from observations, especially when dealing with data of diverse provenance, through the use of feedback mechanisms as described it should be possible to provide data of a sufficient quality for scientific and administrative use. Due to the plausibility information provided for each species identification as well as the reliability ratings attached to each data provided, the end user of the data can filter the available data to assure the necessary level of data quality is provided.

\section{$4 \quad$ Data and Information Models}

\subsection{Standards Based Data Models}

There are several international standards for the structuring, storage and sharing of biodiversity data available; most of these stem from TDWG Biodiversity Information Systems [6], a standards organization specialized in the provision of standards pertaining to biodiversity. The two TDWG standards pertaining to biodiversity occurrence records are:

- Darwin Core (DwC): The Darwin Core is body of standards. The Darwin Core is primarily based on taxa, their occurrence in nature as documented by observations, specimens, and samples, and related information.

- Access to Biological Collections Data (ABCD): The ABCD Schema is a comprehensive standard for the access to and exchange of data about specimens and observations (a.k.a. primary biodiversity data). The ABCD Schema attempts to be comprehensive and highly structured, supporting data from a wide variety of databases.

While these standards have served as an inspiration for the work in ENVIROFI, they have several drawbacks. The following deficits guided us in our decision not to utilize these existing standards:

- ISO Compatibility: The TDWG standards are not compatible with Geography Markup Language (GML) [7] and the wider family of ISO spatial standards mostly defined by the Open Geospatial Consortium (OGC) [8]. As these standards form the backbone of most modern environmental data standards, specifically those defined in the implementation of the INSPIRE Directive (2007/2/EC) [9] in Europe, ISO compliance was deemed essential.

- Lack of Flexibility: while the ISO standards for observational data are very generically defined to guarantee a high level of flexibility, TDWG standards are usually custom tailored to a specific field. The following areas required higher flexibility than the TDWG standards:

- Provision of Multiple Identifications: for the various quality assurance mechanisms described above, it is necessary to be able to provide multiple identifications with integrated plausibility levels for individual biodiversity occurrence records: 
- Dynamic Definition of Properties: due to the differing user groups to be served by the ENVIROFI Biodiversity Application, different observational parameters describing different properties observed on an individual must be supported.

Based on these considerations, the observational data model defined within EN ISO 19156 Observations \& Measurements (O\&M) was selected as the basis for this work. This data model foresees dynamic parameterization of the properties provided, while the TDWG standards would require definition of specialized schemata for this purpose. In addition, as the identification of an individual is seen as an observation on this individual, multiple identifications for one individual can be provided as differing observations on this individual.

The core of the O\&M Schema is the Observation class, which provides a result (association Range) of a specified property (association Phenomenon) determined through the application of a specified procedure (association ProcessUsed) on an explicit object (association Domain) together with relevant metadata such as observation and result times or responsible parties.

The object the properties are being ascertained on, the target of the Domain association has the role featureOfInterest (FoI) for the Observation. Special care must be taken in modeling the FoI, as often properties that should be the result of an observation on the FoI are modeled as explicit attributes of the FoI. An example is the species identification, which is often considered to be an integral part of the object being observed; however, as the identification initially assigned to this individual is often incorrect, it must be seen as an observation on the individual. We have chosen to refer to the FoI defining the individual the observations are being made on as a Species Point (SP), and relegating all further information to the status of observations on this SP.

The following properties have been identified as relevant to tree survey:

Table 1. Tree Survey Properties

\section{Inventory}

Tree number

Identification

Location

Species Identification

Relative Position

Geographic Coordinates

\section{Length Properties}

Breast Height Diameter (BHD)

Tree Height

Crowning Height

Deadwood Height

Crown Diameter

\section{Classifications}

Social Position (Kraft)

Status

Tree Status Forest Inventory

Tree Status Site Inventory

Barkbeetle Infestation

Wind Damage

Light Changes

Tree damage (1-n)

\section{Comments}

Comment 


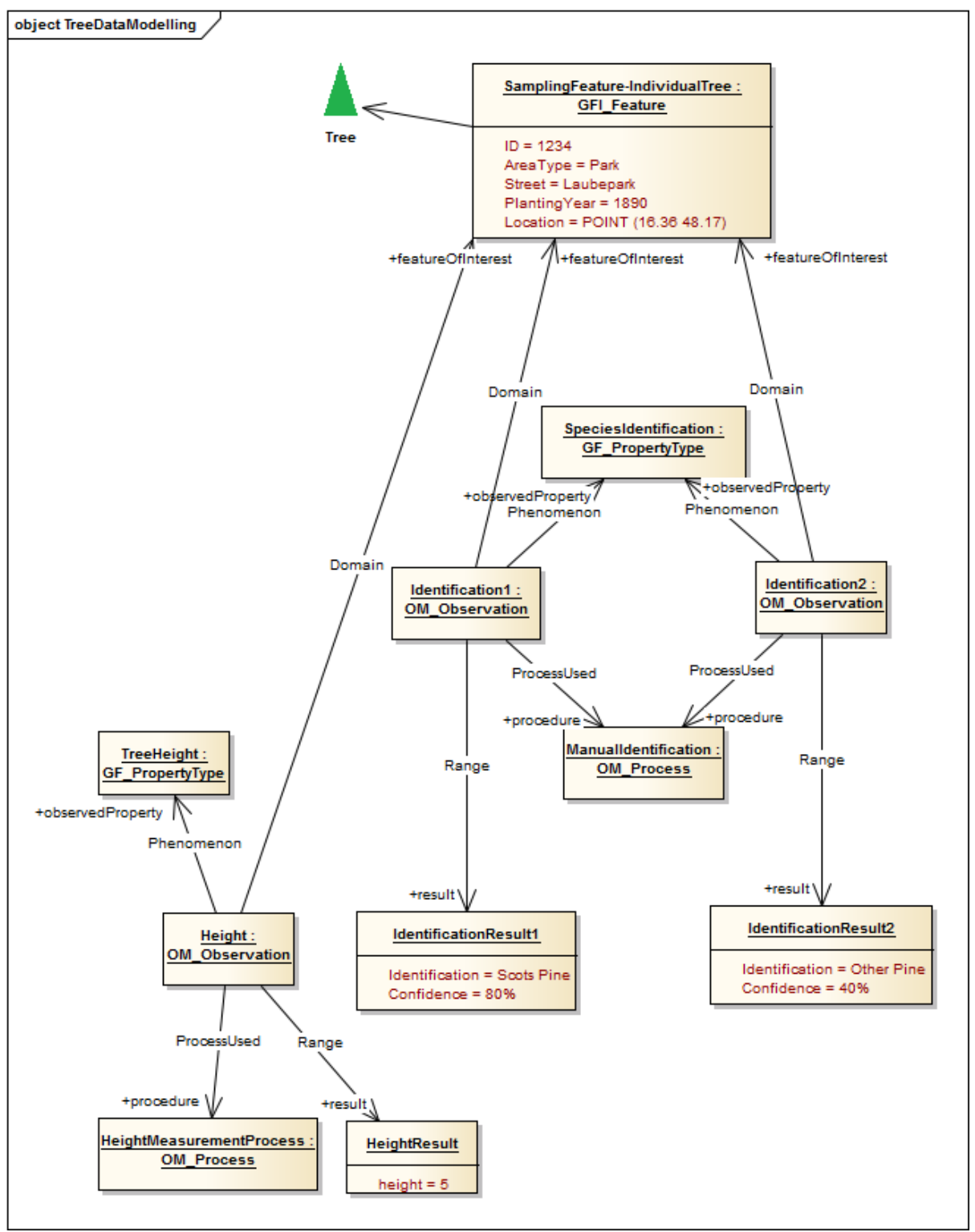

Fig. 1. Individual Species Point with Multiple Identifications

As these properties are dynamically assigned, adding new properties to the system is only a matter of configuration; new parameters can be easily extended. The same is true for the procedure, which is the target of the observation association ProcessUsed; various procedures can be dynamically defined and referred to by observations.

In the example below we provide a simplified sketch of the ENVIROFI Biodiversity data model, based on the O\&M schema. In this example we show how an individual, 
in this case an individual tree in a forest, can have two conflicting identifications with different levels of plausibility as expressed by the confidence attribute in the result.

\subsection{Semantic Backbone}

A recurring problem with the analysis of biodiversity data stems from inconsistencies in the nomenclature used for the identification of species and habitats. Historically, different names have been established for the same concept, the same name been reused for different concepts, or the primary concepts used to describe organisms modified over time. In order to overcome this problem, various projects such as ALTER-Net [10] or SEEK [11] and SONet [12] have proposed the use of ontologies for structuring environmental data.

Within ENVIROFI, it was proposed to utilize the power the TaxMeOn Species Ontology [13] developed of ontologies for the following purposes:

- Structuring and storage of taxonomic information. This should include both scientific and common names

- Storing additional species information as required for context aware quality assurance (i.e. biogeographical regions of occurrence, types often wrongly identified)

- Semantic mapping between species lists (both common and scientific species names from national lists)

The Taxonomic Meta-Ontology (TaxMeOn) is for managing the scientific and vernacular names of organisms. The model consists of three parts that serve different purposes and can be used independently, too. Our focus is here in species lists which forms vary from a simple name list to more information containing list for a professional use.

TaxMeOn defines classes and properties that can be used for building name ontologies for creating semantic metadata for describing observational data. The model is based on $\mathrm{RDF}(\mathrm{S})$ and some features of the OWL, and it contains 42 classes (excl. subclasses of the taxonomic ranks) and 36 properties. The key classes for managing biological names are the following:

1. A scientific name which is binominal and consists of a genus name and a species name. In the Linnaean name system taxa are organized hierarchically (a species, genus, family etc.). The class TaxonInChecklist expresses a scientific name and the hierarchy is expressed using the relation isPartOfHigherTaxon.

2. An author name of a scientific name is a reference to the original publication where the original description of a taxon was first published. The scientific name authorship is expressed using the relation hasScientificNameAuthorship. The author names are often abbreviated, for example Linnaeus may be referred to L. or Linn. The model supports abbreviations. The usage of a reference to an original publication is limited to biology and regulated by the nomenclatural codes, and it differs from the ordinary way of referring to a scientific publication.

3. A reference consists of three subclasses: a checklist, a scientific publication and other source. For instance, the latter can be used if an unofficial note needs to be 
referred to. The class Reference allows associating scientific publication to a name and specifying the checklist that is used.

4. The class Nomenclatural code specifies the set of rules that are applied to a scientific name. The nomenclature of plants and animals differ from each other.

5. According to the nomenclatures a scientific name can have different statuses, for example the status of a scientific name can be rejected.

6. Taxonomic status indicates the current state of a name, for example a name can be a valid name or a synonym of a valid name (the subclass of TaxonomicStatus).

7. The scientific names are easily misspelled and incorrect spellings have often spread in literature. The SpellingStatus is used for indicating the orthographic status of a name, e.g. misspelling.

8. The VernacularName is a class for the common names of organisms in different languages. It is supported to specify a name as an accepted name or an alternative name.

9. Taxonomic rank expresses the hierarchal level of a taxon (a species, a genus, a family etc.; subclasses of the TaxonomicRank).

By providing species identifications through reference to the ontology instead of textual entries, clarity can be achieved in the explicit meaning of the identification. The additional information available to the individual species can be utilizes for context aware quality assurance, provision of additional information on specific species as well as support in merging species occurrence data stemming from other sources.

\section{Conclusions}

In order to meet the requirements for observational biodiversity data, new sources of data must be enabled. For this purpose, new tools will be required; in order to effectively implement such tools, standardized building blocks such as the enablers defined by the FI would be very valuable. Thus, it is of utmost urgency that the requirements posed by the biodiversity sector are clearly structured and made available for implementation of environmental enablers within the FI.

The ENVIROFI project has gone to great lengths so provide these requirements for various environmental domains; of specific relevant to this paper being the biodiversity domain. We hope to successfully contribute to the future of e-Environment in this manner.

The ideas discussed in this paper were also partially developed and tested in the field. More technical information on the overall application architecture and on the specific enablers facilitating the development of FI-enabled VGI applications can be found in [14] and [15] respectively.

Acknowledgements. We thank the ENVIROFI (FP7 - 284898) project consortium for the lively discussions we had as well as the EC for providing the necessary funding for this work. This paper is based on our common findings within the ENVIROFI Project. 


\section{References}

1. European Future Internet Portal, http: / /www. future-internet. eu /

2. Official Homepage of NatureGate, http: / /www. Iuontoportti.com/suomi/en/tekijat/

3. Official Homepage of Laboratoire Informatique \& Systématique, http: / /www. infosyslab.fr/

4. Official Homepage of Laboratoire Informatique \& Systématique Identification Key Software, http: / /www. identificationkey.fr/

5. Official Homepage of the Structured Descriptive Data (SDD) TDWG Standard, http: / / www . tdwg .org/standards/116/

6. Official Homepage of TDWG Biodiversity Information Standards, http: / /www. tdwg.org/

7. Official Homepage for GML, http: / / www . opengeospatial .org/standards/gml/

8. Official Homepage for OGC, http: / /www. opengeospatial.org/

9. INSPIRE Directive Legal Text, http://eur-lex.europa.eu/LexUriServ/ LexUriServ.do?uri=OJ :L:2007:108:0001:0014:EN:PDF

10. Official Homepage for the ALTER-Net Project, http: / / www .alter-net. info/

11. Official Homepage for the SEEK Project, http: / / seek. ecoinformatics.org/

12. Official Homepage for the SONet Project, https: //sonet.ecoinformatics.org/

13. Tuominen, J., Laurenne, N., Hyvönen, E.: Biological Names and Taxonomies on the Semantic Web - Managing the Change in Scientific Conception. In: Antoniou, G., Grobelnik, M., Simperl, E., Parsia, B., Plexousakis, D., De Leenheer, P., Pan, J. (eds.) ESWC 2011, Part II. LNCS, vol. 6644, pp. 255-269. Springer, Heidelberg (2011)

14. Usländer, T., Berre, A., Granell, C., Havlik, D., Lorenzo, J., Sabeur, Z., Modafferi, S.: The Future Internet Enablement of the Environment Information Space. In: Hřebíček, J., et al. (eds.) ISESS 2013. IFIP AICT, vol. 413, Springer, Heidelberg (2013)

15. Havlik, D., Egly, M., Huber, H., Kutschera, P., Falgenhauer, M., Cizek, M.: Robust and trusted crowd-sourcing and crowd-tasking in the Future Internet. In: Hřebíček, J., et al. (eds.) ISESS 2013. IFIP AICT, vol. 413, Springer, Heidelberg (2013) 